\title{
Spatial Correlation of Regional Economic Growth and Regional Coordinated Development Research -----An Empirical Study of Sichuan Province in China
}

\author{
Fengrong Liu ${ }^{1}$, Zhengxia Tang ${ }^{2} \&$ Lapo $\mathrm{Hou}^{3}$ \\ ${ }^{1}$ Center for China Fiscal Development, Central University of Finance and Economics, Beijing, China \\ ${ }^{2}$ School of Economics, Central University of Finance and Economics, Beijing, China \\ ${ }^{3}$ Institute of Yi Language and Culture, Xichang University, Xichang, China \\ Correspondence: Zhengxia Tang, School of Economics, Central University of Finance and Economics, Beijing, \\ China. E-mail: tangzhengxia1988@163.com
}

Received: October 22, 2018

Accepted: November 13, 2018

Online Published: December 2, 2018

doi:10.5539/ijef.v11n1p8

URL: https://doi.org/10.5539/ijef.v11n1p8

\begin{abstract}
Regional economic growth is spatially correlated. With regard to spatial correlation mechanism and characteristics, Network Analysis Method is applied in this paper to discuss the interactions and paths of regional economic development. An empirical study is thus carried out on 18 cities and 3 autonomous prefectures of Sichuan Province to measure their economic network connections and features. We draw main conclusions from this study: (1) the economic network of Sichuan Province shows a typical Core-Periphery structure with Chengdu, the capital city, in the center; (2) geographic location as well as factors such as industrial distributions impose impacts on the formation of the network structure. Finally, based on qualitative evidence and theory discussion, we come up with several suggestions to the coordinated economic development of Sichuan Province.
\end{abstract}

Keywords: economic growth, spatial correlation, coordinated development, social network analysis

\section{Introduction}

Under the background of globalization and regional economic integration, regions have become open complexities rather than closed isolations. Activities between regions contribute to the formation of network relationships. Isard (1960) holds that the real meaning of regional science is the focus on spatial dimensions, and correlations exist in interregional economic growth. Space correlation is the essential feature of geographic phenomenon or process (Ma, 2007). Heavier linkage of regional economic activities emerges along with the infrastructure construction and Internet spreading. Research on spatial correlation is of vital importance for its function to detect principals and explanations of social and economic behaviors (Wei, 2011).

Based on Tobler's First Law of Geography, researchers have been developing the spatial economics especially when applying computer science to the study of spatial correlations. Anselin and Getis (1992) propose that the smaller the spatial units involved in the study, the more possibility that the units are spatially correlated. Proponents of New Economic Geography (NEG) lay huge emphasis on spatial spillover effects, however, the method mainly establishes spatial weight according to distance and adjacency relations while it lacks the capacity to explain economic relationships between nonadjacent regions.

Another method, Social Network Analysis (SNA) of spatial correlations, not only discusses whether regions are adjacent (adjacency relation) but also effectively analyzes the associations between regions with their remote but correlated counterparts. Economic activities are thus abstracted as spatial relationships. Social Network Analysis is a well constructed tool to solve problems of regional development (Kahler \& Lake, 2009) and better reflects regional coordinated development level when applied in the research (Liu, 2014). By introducing complex network theory and method into regional economics, researchers develop the new field which is regional economic network study to explain regional economic phenomenon and principals (Sang \& Qin, 2014). Two or more regions form a network relation through cooperation and relations further establish network structures (Ma, 2014). Infrastructures are seen as substantial carriers for spatial correlations like blood vessels inside human bodies and Internet between computers (Yao, 2003). Labors, capital, materials and information carried by the 
social network are the signals of spatial correlation. Dai (2006) also confirms that transportation and communication are importment parts of regional economic network. Social Network Analysis provides the network structure study with a delicate tool, and more direct and significant measure results with the application of software (Fang, 2013). Li et al. (2014) simulate social network using Social Analysis Method after they detect dynamic correlations between regions using non-structural Vector Autoregressive Model (VAR). Their research method turns out to be valid when describing spatial impact on economic growth.

Recently, Sichuan Province has been playing a leading role in the development of West China. It creates up to $10.8 \%$ of domestic economy increase on average annually from 2011 to 2015 (the 12th five-year planning of China). Meanwhile, rates of increase vary largely of the cities inside the province. In 2015, GDP of Chengdu (the capital city) is 3010.31 billion yuan while the amount is about merely 21.76 billion yuan in Ganzi. Per capita statistics show more evidence. GDP per capita in Panzhihua is 75.10 thousand yuan, which is approximately five times the number of Bazhong (15.10 thousand yuan). What are the reasons of such disparities between the cities of Sichuan Province? The factors can be complicated including history and location, nevertheless, market and administrative division are two main forces that cannot be neglected. On the one hand, the flow of resources between its cities enforces the competitive power of Sichuan Province, but on the other hand, resulting of administrative division reasons, different endowments still contribute to the development disparity. Globalization and regional economy integration are also similar examples of the interregional economic activities' impact on economic growth. In China, government has already implemented strategies like the Belt and Road, Yangtze River Economic Zone, etc. to coordinate regional development.

In this paper, we introduce Social Network Analysis and VAR model to measure dynamic spatial correlation and economic growth relationship between cities in Sichuan Province. Part 2 contains the description of the theory, model and method. Part 3 is an empirical analysis of economic network of cities in Sichuan Province. Part 4 is discussion and comparison on empirical results. And the last part is the conclusions and suggestions.

\section{The Theory, Model and Method}

\subsection{Network Analysis Method}

Economists get to realize that an overall network structure formed by fraction interactions exists in the economic system, which is dominant in the process and evolution of the system (Zhou \& Xu, 2006). Network Analysis essentially focuses on correlations between individuals and their connection approaches to describe the general structure and characteristics. Suppose there are limited numbers of cities in a regional economic system, correlations will spread from nearby neighbors to spatially far regions. Cities are the nodes in the network. Regional resources exchange and move will generate correlations for their spatial relationship which are called ties. Nodes and ties further establish the spatial association network for regional economic growth. Analysis of the spatial network's topology structure contributes to the study of features and function revolution.

\subsubsection{Network Density}

Network density $\left(D_{n}\right)$ is the indicator to the density of correlations between regions in a network. The more the quantity of connections and frequency of resource exchange, the larger network density will be. Liu (2006) identifies network density as the ratio of factual number of ties to the maximum possible ties the network can own. Therefore, density value ranges from 0 to 1 : the value of a totally isolated city is 0 while the value is 1 for a completely connected network. The number of the regions is set to be $\mathrm{N}$ in a network so that maximum correlation number in a spatial network is $N \times(N-1)$. And the current correlation number in a network is set to be $\mathrm{L}$ so that network density $\left(D_{n}\right)$ is expressed as $D_{n}=L /[N \times(N-1)]$.

\subsubsection{Clustering Coefficient}

Clustering Coefficient is a reflection of whether the nodes correlate with other nearby nodes. A triplet is consisted of three nodes that are connected by either two or three undirected ties. It can be explained in the spatial economic network that whether two cities correlate to a certain city also have connections. The nodes clustering coefficient can be expressed as $C_{i}=\frac{1}{k_{i}\left(k_{i}-1\right)} \sum_{j=k}^{n} a_{i j} a_{j k} a_{i j}$. It is the ratio of the number of closed

triples to number of all triples. Network clustering coefficient is defined as $\mathrm{C}=\frac{1}{N} \sum_{i} C_{i}$ based on nodes coefficient, which value represents average correlation value of the network. 


\subsubsection{Centrality}

Centrality measures the status and role of each region in a regional economic network. A city with more centrality value means its tighter correlation with others and more influential capacity to others. There are 3 main measure methods: potint centrality, betweenness centrality and closeness centrality.

Point centrality is the ratio of direct links to a certain region to possible maximum links (Liu, 2006). A city with a lot spatial economic correlations with other cities is generally the core of the network. Point centrality includes Degree Centrality and NrmDegree, we only apply NrmDegree in this paper which is in a standardization form in consideration of further comparisons. The NrmDegree of City A is set to be $D_{u}=\left(\right.$ Outdegree $_{A}+$ Indegree $\left._{A}\right) /$ $2(n-1)$, in which $n$ is the number of the cities in the network. Whether the core point exists is not discussed because point centrality only reflects the relationship between a point with other points in a certain area.

Betweenness centrality is a relative concept first put up by Linton Freeman, which can measure the role a city plays as a bridge between other two cities. Cities that have a high probability to appear in arbitrary paths between two other cities get a high betweenness centrality (Freeman, 1979). The shortest path between City J and City $\mathrm{K}$ is $G_{j k}$, and a City I acts as a economic bridge between them. The possibility of the existence of City I is marked $P_{j k(i)}$. The number of the paths between City $\mathrm{J}$ and City K that go through City I is set to be $G_{j k(i)}$. We get the betweenness centrality as $P_{J k(i)}=G_{j k(i)} / G_{j k}$.

Closeness centrality indicates that resources in a city with high closeness centrality travel to other cities faster than others, which represents its important value in the economic exchange activities. Suppose there are $\mathrm{n}$ nodes in the economic network, then the closeness centrality of City A can be expressed as

$$
C_{C}(A)^{-1}=\sum_{j \neq i}^{N} d_{i j} .
$$

\subsection{VAR Spatial Correlation Model of Regional Economic Development}

Network Analysis is useful in the discussion of spatial relationships as well as the economy gaps. To establish an econometric model of spatial correlation networks, Sims (1990) comes up with non-structural Vector Autoregressive Model (VAR). It regards each variable as endogenous to avoid the lagged virable regression problem. Suppose a VAR expression with two variations

$$
\begin{gathered}
{\left[\begin{array}{l}
\mathrm{y}_{1 t} \\
y_{2 t}
\end{array}\right]=\left[\begin{array}{l}
c_{1} \\
c_{2}
\end{array}\right]+\left[\begin{array}{l}
\beta_{11} \beta_{12} \\
\beta_{21} \beta_{22}
\end{array}\right]\left[\begin{array}{l}
y_{1, t-1} \\
y_{2, t-2}
\end{array}\right]+\left[\begin{array}{l}
u_{1 t} \\
u_{2 t}
\end{array}\right]} \\
u_{1 t}, u_{2 t} \sim \operatorname{IID}\left(0, \sigma^{2}\right), \operatorname{Cov}\left(u_{1 t}, u_{2 t}\right)=0 \\
\text { if we set } Y_{\mathrm{t}}=\left[\begin{array}{l}
\mathrm{y}_{1 t} \\
y_{2 t}
\end{array}\right], C=\left[\begin{array}{l}
c_{1} \\
c_{2}
\end{array}\right], \Gamma=\left[\begin{array}{l}
\beta_{11} \beta_{12} \\
\beta_{21} \beta_{22}
\end{array}\right], u_{t}=\left[\begin{array}{l}
u_{1 t} \\
u_{2 t}
\end{array}\right] \\
\text { then, } Y_{\mathrm{t}}=C_{t}+\Gamma Y_{\mathrm{t}-1}+u_{t}
\end{gathered}
$$

With this VAR model, if a correlation coefficient (City A and B) passes Granger causality test, dynamic correlation exists between the economic growth of City A and City B. Meanwhile, a line or a path is there between these two cities in the network graph. And so on, other spatial correlations can be tested between any two cities to form the final network.

\subsection{Coordination of Regional Development}

Regional coordinated development has been the guide direction of regional development in China (Yang, 2016). Qin (1997) considers that regional coordinated development is a process in which activities and reliances between regions are getting more to achieve the final balanced sustainable status. The concept comprises two meanings. First, regional coordinated development is based on interregional labor division and cooperation. Division and cooperation involve resources, products, technology and labor division, which takes comparative advantage to promote efficiency and profits in the whole area. The various endowment conditions of different regions is fundamental to the division and cooperation. Regions have got their own comparative advantages which can be taken in priority to gain economies of scale and better overall competitive power. Next, disparities can be narrowed to a reasonable range in the correlation of the region development to obtain sustainable development (Hu, 2016). Solid network relationship rather than fractional links is more likely to benefit regional coordinated development.

\section{Empirical Study on Spatial Correlations of Economic Growth: Cities in Sichuan Province}

\subsection{Data}

Sichuan Province is located in Southwest China and consists of 18 cities and 3 autonomous prefectures. The 21 
regions are developing at different stages, such as Chengdu, the best one, and poor cities like Liangshan autonomous prefecture, Aba autonomous prefecture, Guangyuan and Bazhong, which are typical examples of extremely poor areas in China

The data used in this paper are GDP per capita (1998-2015) of 18 cities and 3 autonomous prefectures in Sichuan Province.

\subsection{Spatial Correlating Network of Economic Growth}

Sichuan Province adjusted its administrative divisions in 1997 and maintains till now so that we collect data from 1998 to analyze the spatial correlation of economic growth. Detrended data are applied into VAR model. 85 correlations pass the Granger causality test and network of city economic growth graph is drawn by PAJEK (Figure 1).

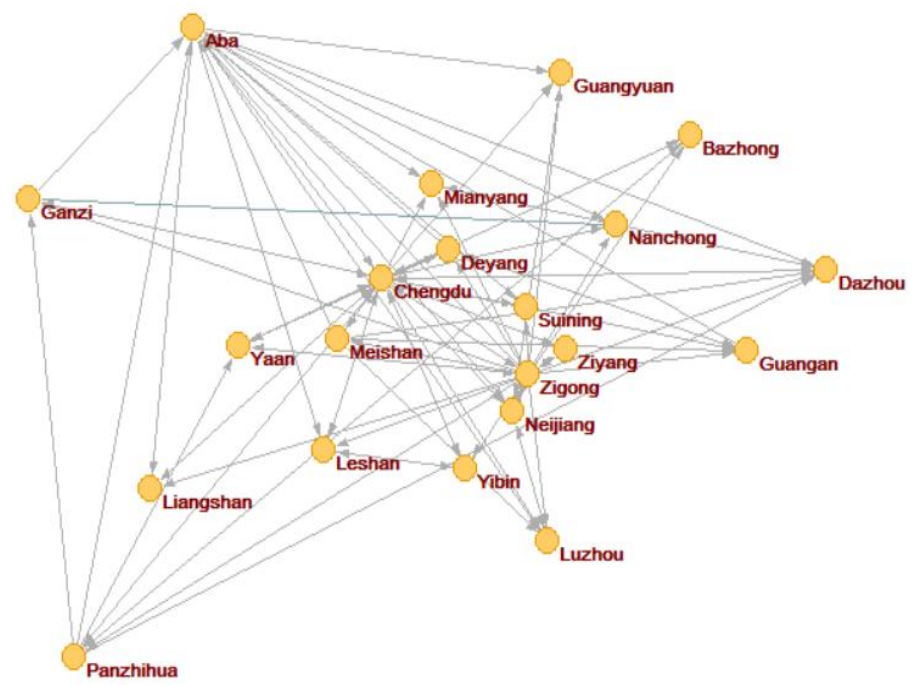

Figure 1. Spatial correlation network of economic growth in Sichuan province

Figure 1 exhibites that the economic correlations are common among 21 prefectures in Sichuan Province although the strength varies. Chengdu is obviously the core of the network and a "Core- Periphery" phenomenon appears. Besides, neighborhood reliance is significant in this network that a lot cities have close relationship with their nearby cities. An outlier, Aba autonomous prefecture, which lies far from the core city both in the real world and the above graph still has many correlations with other cities. The reason for that is Aba enjoys abundant tourism resources to correlate with other cities and has established its own economic pattern because of the early development. Other cities like Ganzi, Liangshan, Ya'an, Guangyuan and Bazhong are not as lucky as Aba and are still at the stage of poor and comparatively closed development.

\subsection{Features of the Spatial Economic Correlation Network}

\subsubsection{Network Density}

Empirical data analysis will be shown below to explain more information apart from Figure 1. According to the result PAJEK gives, the maximum correlation number is 420 , however, the number in the real world is only 85 . Network density can thus be calculated, about 0.20 , which is low. Fortunately, the fact that any two cities can be correlated by another city in Sichuan Province eases the difficulty in regional economic coordination.

\subsubsection{Clustering Coefficient}

Clustering coefficient is displayed in the last column of Table 1. Clustering coefficient of the city economic growth network in Sichuan Province ranges from 0.002439 to 1. Chengdu and Zigong shows the highest value 1, which means their correlation degrees with other regions inside the province are high. The next is Aba, with the coefficient 0.588235 due to reasons discussed formerly. Other cities show very low value less than 0.1 .

\subsubsection{Centrality}

In Table 1, the second, third and fourth column respectively represent potint centrality, betweenness centrality and closeness centrality. No doubt Chengdu is the core of the province with all the highest 3 kinds of centrality 
value and strongest correlations with other 20 prefectures. The second prize, Zigong, also enjoys a lot of correlations with other cities. And the third one is Aba as a result of its resources advantage and economic growth pattern. Aba's mature tourism industry is supported by outside correlations, which also proves network analysis fit the reality and offers references to other remote cities to develop their own economy.

Upon the analysis of network features, spatial economic correlations in Sichuan Province show typical "CorePeriphery" structure. Core cities like Chengdu and Zigong have spatial correlations with others while the periphery cities show less correlations. The correlation degree generally decays from core to periphery.

Table 1. Spatial network characteristics of economic growth in Sichuan province

\begin{tabular}{lccccc}
\hline District & Point Centrality & Betweenness Centrality & Closeness Centrality & Structural Holes & Clustering Coefficient \\
\hline Chengdu & 32 & 0.470175 & 1 & 0.200737 & 1 \\
Zigong & 24 & 0.213158 & 1 & 0.24167 & 1 \\
Panzhihua & 8 & 0 & 0.606061 & 0.406269 & 0.090909 \\
Luzhou & 6 & 0.000877 & 0.571429 & 0.414901 & 0.041139 \\
Deyang & 7 & 0.012719 & 0.571429 & 0.408904 & 0.038462 \\
Mianyang & 5 & 0 & 0.555556 & 0.451446 & 0.0225 \\
Guangyuan & 4 & 0 & 0.555556 & 0.41985 & 0.022222 \\
Suining & 3 & 0 & 0.540541 & 0.435434 & 0.009146 \\
Neijiang & 7 & 0.101974 & 0.571429 & 0.405997 & 0.035256 \\
Leshan & 6 & 0.002193 & 0.555556 & 0.488054 & 0.020253 \\
Nanchong & 3 & 0 & 0.540541 & 0.435434 & 0.009146 \\
Yibin & 5 & 0 & 0.540541 & 0.575347 & 0.009375 \\
Guang'an & 6 & 0.001316 & 0.571429 & 0.417178 & 0.041139 \\
Dachuan & 8 & 0.051754 & 0.588235 & 0.379925 & 0.062338 \\
Ya'an & 6 & 0.003289 & 0.555556 & 0.443021 & 0.020253 \\
Bazhong & 4 & 0 & 0.540541 & 0.523003 & 0.009259 \\
Meishan & 7 & 0 & 0.588235 & 0.352992 & 0.057692 \\
Ziyang & 3 & 0 & 0.526316 & 0.623891 & 0.002439 \\
Aba & 17 & 0.076754 & 0.833333 & 0.277731 & 0.588235 \\
Ganzi & 5 & 0 & 0.555556 & 0.435622 & 0.0225 \\
Liangshan & 4 & 0 & 0.540541 & 0.475429 & 0.009259 \\
\hline
\end{tabular}

\section{Influencing Factors}

First of all, network characteristics indicators are displayed in Figure 2, including potint centrality, betweenness centrality, closeness centrality and clustering coefficient. Chengdu, as always, is the core city with tight economic relations to other cities. Next, it comes to Zigong and Aba, the second echelon. Then, Suining, Neijiang, Yibin, Luzhou, Panzhihua etc. mostly locates in the southeastern areas except for Panzhihua, a city lies in the southwest far from these cities. Figure 2 further implies that economic correlations not only exist in neighborhood relations but also in faraway cities. In this case, the correlation appears because of industrial development. Panzhihua is a city standing on steel industry which requires support from the outside and exports products to other cities. The last echelon, Ganzi, Liangshan, Guangyuan, Bazhong is in the western and northeastern areas of Sichuan Province.

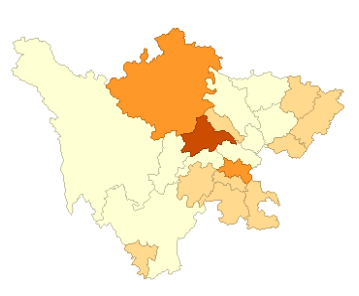

a. Point Centrality

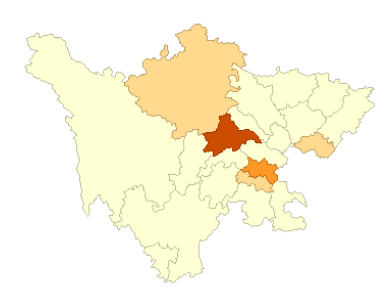

b. Betweenness Centrality

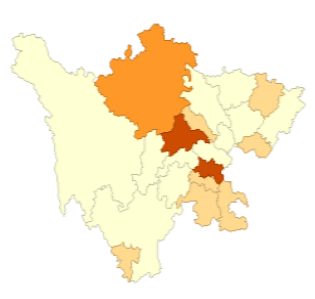

c. Closeness Centrality

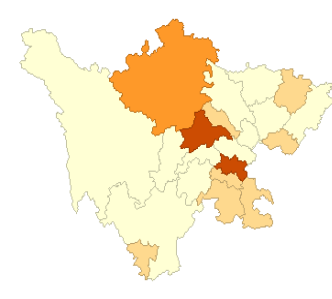

f. Clustering Coefficient

Figure 2. Centrality analysis of spatial correlation in Sichuan province 
Based on the findings that industry features contribute to the connections, we introduce more factors in the analysis. Figure 3 shows possible influential factors. Secondary industry proportion, tertiary industry proportion, quantity of colleges and universities, roads density, number of enterprises etc. are factors correlated strongly to the spatial economic growth.

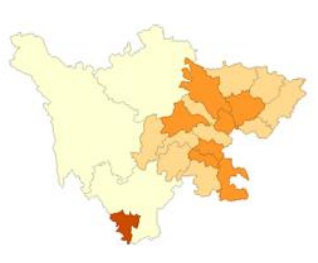

a. Secondary Industry

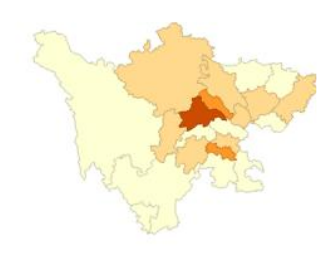

b. Tertiary Industry Proportion

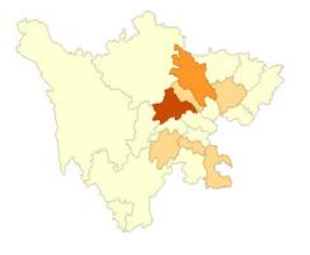

c. Quantity of Colleges and Universities

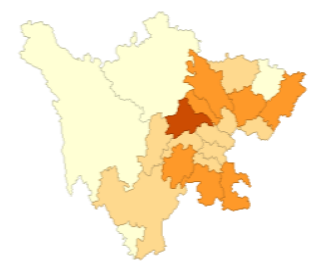

g. Annual Bank Savings

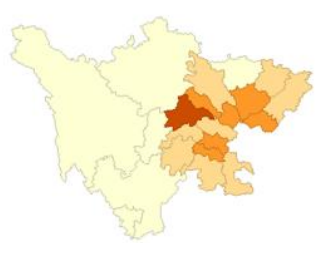

d. Roads Density

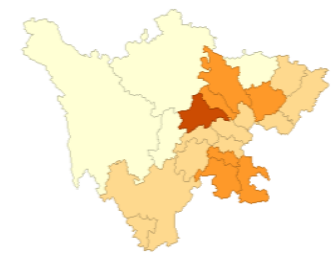

e. Number of Enterprises

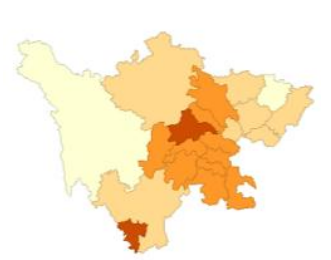

f. Economic Value Added of Private Economy (Per Capita)

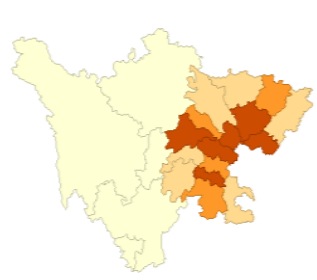

h. Population Density

Figure 3. Influencing factors on spatial correlation

\section{Suggestions}

Spatial correlations are common in the economic development between cities in Sichuan Province, however, the correlations seem to be relatively weak. Under the background of regional coordinated development in China, this paper proposes several suggestions to the development in Sichuan Province:

Accessiblity between regions should be enhanced. Roads are important infrastructures in the economic network. Current roads network in Sichuan Province still needs to be constructed, for roads between Chengdu and periphery regions are in low grade and roads between periphery cities are not adequate. Resources exchange and move can be limited by the roads conditions. Roads density inside the province is still low. Roads construction is necessary to form economic network and exert radiating function of core cities.

Exploiting comparative advantages of each region. The examples of Aba and Panzhihua tell the possibility that comparative advantage can generate economic correlations and better development. Similar regions, Ganzi and Liangshan may replicate the same path to develop tertiary industry with their ample tourism endowments. Regions, especially small cities are not necessarily developed from primary industry to tertiary industry step by step. Their own advantage should be discovered and expanded according to their characteristics.

More attention is required to the development of southeastern cities in Sichuan Province. The direct distance between cities in the southeast are short and the closeness is a basis for coordination. These cities are capable of accepting the industrial transfer from Chengdu or even South China.

\section{References}

Chen, Q. (2013). Advanced Econometrics and Stata Application. Beijing: Higher Education Press.

Dai, H. W. (2006). Regional Economy Coordination and County Economy Development. Daily Economic Newspaper in Hebei.

Fang, D. C., \& Zhou, Z. R. (2013). Research on the Structure of Economic Ties in Cities in Anhui Province: Based on the Social Network Analysis. East China Economic Management, 1, 8-23.

Freeman, L. C. (1979). Centrality in Social Networks: Conceptual Clarification. Social Networks, 220-225. 
Guo, L., \& Xu, X. M. (2006). Complex Network. Shanghai: Shanghai Scientific \& Technical Publishers.

Hu, J., \& Chen, J. M. (2016, June). Study on the Coordinated Development of Economy of Liangshan County Economy Based on Difference. Guizhou Ethnic Studies. https://doi.org/10.13965/j.cnki.gzmzyj10026959.2016.06.037

Isard, W. (1960). Methods of Regional Analysis: An Introduction to Regional Science. Cambridge, Massachusetts: The MIT Press.

Kahler, M., \& Lake, D. A. (2009). Economic integration and global governance: Why so little supranationalism? In W. Mattli, \& N. Woods (Eds.), The politics of global regulation (pp. 242-277). Princeton University Press,Princeton.

Li, J., Chen, S., \& Wang, G. H. (2014, October). Study on the Spatial Correlation and Expanlation of Regional Economic Growth in China. Economic Research Journal.

Liu, B. L., Su, H., \& Zhang, W. T. (2014). Regional Coordinated Development Analysis Based on Complex Network. Journal of Commercial Economics, 22.

Liu, J. (2006). Introduction to Social Network Analysis. Beijing: Social Sciences Academic Press.

Ma, J., Suo, L. M., \& Chen, B. (2014). From Collaborative Zone to Collaborative Network: Structure, Pathway and Evolution—-The Analysis 191 Intergovernment Agreements in "9+2" Zone. China Soft Science, 12.

Ma, R. H., \& Pu, Y. X. (2007). Discoveries of GIS Spatial Correlation. Beijing: Science Press .

Qin, C. L. (1997). Research on disparities of China's regional economy. Beijing: China Economic Publishing House.

Sang, M. L., \& Tan, C. L. (2014). A New Trend In Regional Economics: Research On Regional Economic Networks. Human Geography, 3, 28-35. https://doi.org/10.13959/j.issn.1003-2398.2014.03.031

Wei, H. K. (2011). Modern Regional Economics. Beijing: Economy \& Management Publishing House.

Yang, K. Z., \& Xue, S. (2002, February). Spatial Complexity: Regional Science in 21th Century. Advances in Earth Science.

Yao, S. M., Wang, C. X., \& Zhu, Z. G. (2003, September). New Research Fields in Urban Geography. Economic Geography. https://doi.org/10.15957/j.cnki.jjdl.2003.05.011

\section{Copyrights}

Copyright for this article is retained by the author(s), with first publication rights granted to the journal.

This is an open-access article distributed under the terms and conditions of the Creative Commons Attribution license (http://creativecommons.org/licenses/by/4.0/). 